\title{
IMPROVEMENT OF AN INEQUALITY OF G. H. HARDY
}

\author{
SAJID IQBAL ${ }^{1}$, KRISTINA KRULIĆ ${ }^{2}$ and JOSIP PEČARIĆ ${ }^{1,3}$
}

\begin{abstract}
In this paper, we give an improvement of an inequality of G. H. Hardy using fractional integrals and fractional derivatives. We also obtain means of Cauchy type and prove their monotonicity.
\end{abstract}

\section{Introduction}

We start with the definition of the Riemann-Liouville fractional integral operator. First, let us recall the well known definitions of Riemann-Liouville fractional integrals, see [11] and [3].

Let $[a, b]$ be a finite interval on real axis $\mathbb{R}$. The Riemann-Liouville fractional integrals $I_{a^{+}}^{\alpha} f$ and $I_{b^{-}}^{\alpha} f$ of order $\alpha>0$ are defined by

and

$$
I_{a^{+}}^{\alpha} f(x)=\frac{1}{\Gamma(\alpha)} \int_{a}^{x} f(y)(x-y)^{\alpha-1} d y,(x>a)
$$

$$
I_{b^{-}}^{\alpha} f(x)=\frac{1}{\Gamma(\alpha)} \int_{x}^{b} f(y)(y-x)^{\alpha-1} d y, \quad(x<b) .
$$

Here $\Gamma(\alpha)$ is the Gamma function. These integrals are called the left-sided and right-sided fractional integrals respectively. The first result yields that fractional integral operators are bounded in $L_{p}(a, b),-\infty<a<b<\infty, 1 \leq p \leq \infty$, that is

$$
\left\|I_{a^{+}}^{\alpha} f\right\|_{p} \leq K\|f\|_{p}, \quad\left\|I_{b^{-}}^{\alpha} f\right\|_{p} \leq K\|f\|_{p}
$$

where

$$
K=\frac{(b-a)^{\alpha}}{\Gamma(\alpha+1)} .
$$

G. H. Hardy proved the inequality (1.1) involving left-sided fractional integral in one of his initial paper, see [6]. The calculation for the constant $K$ is hidden inside the proof.

Corresponding author: Sajid Iqbal.

2010 Mathematics Subject Classification. 26D15, 26D10, 26 A33.

Key words and phrases. Convex function, kernel, fractional derivative, fractional integrals, means. 
In this paper, we give improvements of Hardy type inequalities given in [8]. We also establish new inequalities involving fractional integrals and fractional derivatives of RiemmanLiouville, Caputo, Canavati, Erdelyi-Kóber and Hadamard-type.

Let $\left(\Omega_{1}, \Sigma_{1}, \mu_{1}\right)$ and $\left(\Omega_{2}, \Sigma_{2}, \mu_{2}\right)$ be measure spaces with $\sigma$-finite measures and $A_{k}$ be an integral operator defined by

$$
A_{k} f(x):=\frac{1}{K(x)} \int_{\Omega_{2}} k(x, y) f(y) d \mu_{2}(y)
$$

where $k: \Omega_{1} \times \Omega_{2} \rightarrow \mathbb{R}$ is measurable and non-negative kernel, $f$ is measurable function on $\Omega_{2}$, and

$$
K(x):=\int_{\Omega_{2}} k(x, y) d \mu_{2}(y), \quad x \in \Omega_{1} .
$$

Throughout the paper, we consider that $K(x)>0$ a.e. on $\Omega_{1}$.

The following theorem is give in [12] (see also [4]).

Theorem 1.1. Let $\left(\Omega_{1}, \Sigma_{1}, \mu_{1}\right)$ and $\left(\Omega_{2}, \Sigma_{2}, \mu_{2}\right)$ be measure spaces with $\sigma$-finite measures, $u$ be a weight function on $\Omega_{1}$, $k$ be a non-negative measurable function on $\Omega_{1} \times \Omega_{2}$, and $K$ be defined on $\Omega_{1}$ by (1.3). Suppose that the function $x \mapsto u(x) \frac{k(x, y)}{K(x)}$ is integrable on $\Omega_{1}$ for each fixed $y \in \Omega_{2}$, and that $v$ is defined on $\Omega_{2}$ by

$$
v(y):=\int_{\Omega_{1}} \frac{u(x) k(x, y)}{K(x)} d \mu_{1}(x)<\infty .
$$

If $\Phi$ is convex on the interval $I \subseteq \mathbb{R}$, then the inequality

$$
\int_{\Omega_{1}} u(x) \Phi\left(A_{k} f(x)\right) d \mu_{1}(x) \leq \int_{\Omega_{2}} v(y) \Phi(f(y)) d \mu_{2}(y),
$$

holds for all measurable functions $f: \Omega_{2} \rightarrow \mathbb{R}$, such that Imf $\subseteq I$, where $A_{k}$ is defined by (1.2).

For reader's convenience, we introduce some necessary notation and recall some basic facts about convex functions, log-convex functions (see e.g. [10], [15]) as well as exponentially convex functions (see e.g [2], [13], [14]).

Definition 1.2. A positive function $f$ is said to be $\operatorname{logarithmically~convex~if~} \log f$ is a convex function on an interval $I \subseteq \mathbb{R}$. For such function $f$, we shortly say $f$ is log-convex. A positive function $f$ is log-convex in the Jensen sense if for each $a, b \in I$

$$
f^{2}\left(\frac{a+b}{2}\right) \leq f(a) f(b)
$$

holds, i.e., if $\log f$ is convex in the Jensen sense. 
The following definition is equivalent to the definition of convex functions.

Definition 1.3 ([16]). Let $I \subseteq \mathbb{R}$ be an interval and $f: I \rightarrow \mathbb{R}$ be convex on $I$. Then for $s_{1}, s_{2}, s_{3} \in I$ such that $s_{1}<s_{2}<s_{3}$, the following inequality holds:

$$
f\left(s_{1}\right)\left(s_{3}-s_{2}\right)+f\left(s_{2}\right)\left(s_{1}-s_{3}\right)+f\left(s_{3}\right)\left(s_{2}-s_{1}\right) \geq 0 .
$$

Function $f$ is log-convex on an interval $I$, if and only if for all $s_{1}, s_{2}, s_{3} \in I, s_{1}<s_{2}<s_{3}$ it holds

$$
\left[f\left(s_{2}\right)\right]^{s_{3}-s_{1}} \leq\left[f\left(s_{1}\right)\right]^{s_{3}-s_{2}}\left[f\left(s_{3}\right)\right]^{s_{2}-s_{1}} .
$$

This lemma help us to prove the monotonicity of means.

Lemma 1.4. Let positive function $f: I \rightarrow \mathbb{R}$ be $\log$-convex and let $a_{1}, a_{2}, b_{1}, b_{2} \in I$ such that $a_{1} \leq b_{1}, a_{2} \leq b_{2}$ and $a_{1} \neq a_{2}, b_{1} \neq b_{2}$. Then the following inequality holds:

$$
\left[\frac{f\left(a_{2}\right)}{f\left(a_{1}\right)}\right]^{\frac{1}{a_{2}-a_{1}}} \leq\left[\frac{f\left(b_{2}\right)}{f\left(b_{1}\right)}\right]^{\frac{1}{b_{2}-b_{1}}} .
$$

Let us recall the following definition of exponentially convex functions and very important proposition.

Definition 1.5. ([13, p.373]) A function $h:(a, b) \rightarrow \mathbb{R}$ is exponentially convex if it is continuous and

$$
\sum_{i, j=1}^{n} a_{i} a_{j} h\left(x_{i}+x_{j}\right) \geq 0,
$$

for all $n \in \mathbb{N}$ and all choices of $a_{i} \in \mathbb{R}, x_{i}+x_{j} \in(a, b), i, j=1, \ldots, n$.

Proposition 1.6 ([7]). Let $h:(a, b) \rightarrow \mathbb{R}$. The following statements are equivalent.

(i) $h$ is exponentially convex.

(ii) $h$ is continuous and

$$
\sum_{i, j=1}^{n} a_{i} a_{j} h\left(\frac{x_{i}+x_{j}}{2}\right) \geq 0,
$$

for every $n \in \mathbb{N}, a_{i} \in(a, b)$ and $x_{i} \in(a, b), 1 \leq i \leq n$.

Every exponentially convex function is log-convex in the Jensen sense, and, being continuous, it is also log-convex function. However, converse is not true: the function $h(x)=e^{x^{3}-x}$ is log-convex on $(0,1)$ but it is not exponentially convex on $(0,1)$. (see [9])

Throughout this paper, all measures are assumed to be positive, all functions are assumed to be positive and measurable and expressions of the form $0 \cdot \infty, \frac{\infty}{\infty}$ and $\frac{0}{0}$ are taken 
to be equal to zero. Moreover, by a weight $u=u(x)$ we mean a nonnegative measurable function on the actual interval or more general set.

The paper is organized in the following way. After introduction, in Section 2, we give the improvement of an inequality of G. H. Hardy related to fractional derivatives and fractional integrals. We obtain new inequalities which involve different fractional integrals and fractional derivatives of Riemman-Liouville, Caputo, Canavati, Erdelyi-Kóber and Hadamard-type. We establish mean value theorems of Cauchy type and means of Cauchy type related to different fractional integrals and fractional derivatives and prove monotonicity of these means.

\section{The main results}

Lemma 2.1. Let $s \in \mathbb{R}, \varphi_{s}: \mathbb{R}^{+} \rightarrow \mathbb{R}$ be a function defined as

$$
\varphi_{s}(x):= \begin{cases}\frac{x^{s}}{s(s-1)}, & s \neq 0,1, \\ -\log x, & s=0, \\ x \log x, & s=1 .\end{cases}
$$

Then $\varphi_{s}$ is strictly convex on $\mathbb{R}^{+}$for each $s \in \mathbb{R}$.

Proof. Since $\varphi_{s}^{\prime \prime}(x)=x^{s-2}>0$ for all $x \in \mathbb{R}^{+}, s \in \mathbb{R}$ therefore $\varphi_{s}$ is strictly convex on $\mathbb{R}^{+}$for each $s \in \mathbb{R}$.

The following theorem is given in [4].

Theorem 2.2. Let the assumption of the Theorem 1.1 be satisfied and $\varphi_{s}$ be defined by (2.1). Let $f$ be a positive function. Then the function $\xi: \mathbb{R} \rightarrow[0, \infty)$ is defined by

$$
\xi(s)=\int_{\Omega_{2}} v(y) \varphi_{s}(f(y)) d \mu_{2}(y)-\int_{\Omega_{1}} u(x) \varphi_{s}\left(A_{k} f(x)\right) d \mu_{1}(x)
$$

is exponentially convex.

The function $\xi$ being exponentially convex is also log - convex function. Then by Definition 1.3 the following inequality holds true:

$$
[\xi(p)]^{q-r} \leq[\xi(q)]^{p-r}[\xi(r)]^{q-p}
$$

for every choice $r, p, q \in \mathbb{R}$, such that $r<p<q$.

We will give some special cases of Theorem 2.2 for different fractional integrals and fractional derivatives to establish new inequalities.

Here, we give a first special case of Theorem 2.2 for the Riemman-Liouville fractional integral. 
Theorem 2.3. Let $s>1, \alpha>0, I_{a^{+}}^{\alpha} f$ denotes the left-sided Riemann-Liouville fractional integral of $f$ and $\xi_{1}: \mathbb{R} \rightarrow[0, \infty)$. Then the following inequality holds true:

$$
\xi_{1}(s) \leq H_{1}(s),
$$

where

$$
\xi_{1}(s)=\frac{1}{s(s-1)}\left[\int_{a}^{b}(b-y)^{\alpha} f^{s}(y) d y-\int_{a}^{b}(x-a)^{\alpha}\left(\frac{\Gamma(\alpha+1)}{(x-a)^{\alpha}} I_{a^{+}}^{\alpha} f(x)\right)^{s} d x\right]
$$

and

$$
H_{1}(s)=\frac{(b-a)^{\alpha(1-s)}}{s(s-1)}\left[(b-a)^{\alpha s} \int_{a}^{b} f^{s}(y) d y-(\Gamma(\alpha+1))^{s} \int_{a}^{b}\left(I_{a^{+}}^{\alpha} f(x)\right)^{s} d x\right] .
$$

Proof. Applying Theorem 2.2 with $\Omega_{1}=\Omega_{2}=(a, b), d \mu_{1}(x)=d x, d \mu_{2}(y)=d y$,

$$
k(x, y)= \begin{cases}\frac{(x-y)^{\alpha-1}}{\Gamma(\alpha)}, & a \leq y \leq x \\ 0, & x<y \leq b\end{cases}
$$

we get that $K(x)=\frac{(x-a)^{\alpha}}{\Gamma(\alpha+1)}$. Then the equation (2.2) becomes

$$
\xi_{1}(s)=\int_{a}^{b} v(y) \phi_{s}(f(y)) d y-\int_{a}^{b} u(x) \phi_{s}\left(\frac{\Gamma(\alpha+1)}{(x-a)^{\alpha}} I_{a^{+}}^{\alpha} f(x)\right) d x .
$$

For particular weight function $u(x)=(x-a)^{\alpha}, x \in(a, b)$ we get $\nu(y)=(b-y)^{\alpha}$ and we take $\phi_{s}(x)=\frac{x^{s}}{s(s-1)}, x \in \mathbb{R}^{+}$. So (2.5) becomes

$$
\begin{aligned}
\xi_{1}(s) & =\frac{1}{s(s-1)}\left[\int_{a}^{b}(b-y)^{\alpha} f^{s}(y) d y-\int_{a}^{b}(x-a)^{\alpha}\left(\frac{\Gamma(\alpha+1)}{(x-a)^{\alpha}} I_{a^{+}}^{\alpha} f(x)\right)^{s} d x\right] \\
& \leq \frac{1}{s(s-1)}\left[(b-a)^{\alpha} \int_{a}^{b} f^{s}(y) d y-(b-a)^{\alpha(1-s)}(\Gamma(\alpha+1))^{s} \int_{a}^{b}\left(I_{a^{+}}^{\alpha} f(x)\right)^{s} d x\right] \\
& =\frac{(b-a)^{\alpha(1-s)}}{s(s-1)}\left[(b-a)^{\alpha s} \int_{a}^{b} f^{s}(y) d y-(\Gamma(\alpha+1))^{s} \int_{a}^{b}\left(I_{a^{+}}^{\alpha} f(x)\right)^{s} d x\right] \\
& =H_{1}(s) .
\end{aligned}
$$

It follows (2.4).

Theorem 2.4. Let $s>1, \alpha>0, I_{b^{-}}^{\alpha} f$ denotes the right-sided Riemann-Liouville fractional integral of $f$ and $\xi_{2}: \mathbb{R} \rightarrow[0, \infty)$. Then the following inequality holds true:

$$
\xi_{2}(s) \leq H_{2}(s),
$$

where

and

$$
\xi_{2}(s)=\frac{1}{s(s-1)}\left[\int_{a}^{b}(y-a)^{\alpha} f^{s}(y) d y-\int_{a}^{b}(b-x)^{\alpha}\left(\frac{\Gamma(\alpha+1)}{(b-x)^{\alpha}} I_{b^{-}}^{\alpha} f(x)\right)^{s} d x\right]
$$

$$
H_{2}(s)=\frac{(b-a)^{\alpha(1-s)}}{s(s-1)}\left[(b-a)^{\alpha s} \int_{a}^{b} f^{s}(y) d y-(\Gamma(\alpha+1))^{s} \int_{a}^{b}\left(I_{b^{-}}^{\alpha} f(x)\right)^{s} d x\right] .
$$


Proof. Similar to the proof of Theorem 2.2.

Next we give result with respect to the generalized Riemann-Liouville fractional derivative. Let us recall the definition, for details see [1, p.448].

We define the generalized Riemann-Liouville fractional derivative of $f$ of order $\alpha>0$ by

$$
D_{a}^{\alpha} f(x)=\frac{1}{\Gamma(n-\alpha)}\left(\frac{d}{d x}\right)^{n} \int_{a}^{x}(x-y)^{n-\alpha-1} f(y) d y,
$$

where $n=[\alpha]+1, x \in[a, b]$.

We say that $f \in L_{1}(a, b) ; a, b \in \mathbb{R}$ has an $L_{\infty}$ fractional derivative $D_{a}^{\alpha} f(\alpha>0)$ in $[a, b]$, iff

(1) $D_{a}^{\alpha-k} f \in C([a, b]), k=1, \ldots, n=[\alpha]+1$

(2) $D_{a}^{\alpha-1} f \in A C([a, b])$, and

(3) $D_{a}^{\alpha} f \in L_{\infty}(a, b)$.

The following lemma help us to prove the next result. For details see [1, p.449] (also see [5]).

Lemma 2.5. Let $\beta>\alpha \geq 0$ and let $f \in L_{1}(a, b)$ have an $L_{\infty}$ fractional derivative $D_{a}^{\beta} f$ in $[a, b]$ and let

$$
D_{a}^{\beta-k} f(a)=0, \quad k=1, \ldots,[\beta]+1 .
$$

Then

$$
D_{a}^{\alpha} f(x)=\frac{1}{\Gamma(\beta-\alpha)} \int_{a}^{x}(x-y)^{\beta-\alpha-1} D_{a}^{\beta} f(y) d y
$$

for all $a \leq x \leq b$.

Theorem 2.6. Let $s>1, \beta>\alpha \geq 0$ and let $f \in L_{1}(a, b)$ have an $L_{\infty}$ fractional derivative $D_{a}^{\beta} f$ in $[a, b]$, and $\xi_{3}: \mathbb{R} \rightarrow[0, \infty)$. Then the following inequality holds true:

$$
\xi_{3}(s) \leq H_{3}(s)
$$

where

$$
\xi_{3}(s)=\frac{1}{s(s-1)}\left[\int_{a}^{b}(b-y)^{\beta-\alpha}\left(D_{a}^{\beta} f(y)\right)^{s} d y-\int_{a}^{b}(x-a)^{\beta-\alpha}\left(\frac{\Gamma(\beta-\alpha+1)}{(x-a)^{\beta-\alpha}}\left(D_{a}^{\alpha} f(x)\right)\right)^{s} d x\right]
$$

and

$$
H_{3}(s)=\frac{(b-a)^{(\beta-\alpha)(1-s)}}{s(s-1)}\left[(b-a)^{(\beta-\alpha) s} \int_{a}^{b}\left(D_{a}^{\beta} f(y)\right)^{s} d y-(\Gamma(\beta-\alpha+1))^{s} \int_{a}^{b}\left(D_{a}^{\alpha} f(x)\right)^{s} d x\right] .
$$

Proof. Applying Theorem 2.2 with $\Omega_{1}=\Omega_{2}=(a, b), d \mu_{1}(x)=d x, d \mu_{2}(y)=d y$,

$$
k(x, y)= \begin{cases}\frac{(x-y)^{\beta-\alpha-1}}{\Gamma(\beta-\alpha)}, & a \leq y \leq x \\ 0, & x<y \leq b\end{cases}
$$


we get that $K(x)=\frac{(x-a)^{\beta-\alpha}}{\Gamma(\beta-\alpha+1)}$. Replace $f$ by $D_{a}^{\beta} f$. Then the equation (2.2) becomes

$$
\xi_{3}(s)=\int_{a}^{b} v(y) \phi_{s}\left(D_{a}^{\beta} f(y)\right) d y-\int_{a}^{b} u(x) \phi_{s}\left(\frac{\Gamma(\beta-\alpha+1)}{(x-a)^{\beta-\alpha}} D_{a}^{\alpha} f(x)\right) d x .
$$

For particular weight function $u(x)=(x-a)^{\beta-\alpha}$, we get $v(y)=(b-y)^{\beta-\alpha}$ and we choose $\phi_{s}(x)=\frac{x^{s}}{s(s-1)}, x \in \mathbb{R}^{+}$. So, (2.8) becomes

$$
\begin{aligned}
\xi_{3}(s) & =\frac{1}{s(s-1)}\left[\int_{a}^{b}(b-y)^{\beta-\alpha}\left(D_{a}^{\beta} f(y)\right)^{s} d y-\int_{a}^{b}(x-a)^{\beta-\alpha}\left(\frac{\Gamma(\beta-\alpha+1)}{(x-a)^{\beta-\alpha}}\left(D_{a}^{\alpha} f(x)\right)\right)^{s} d x\right] \\
& \leq \frac{1}{s(s-1)}\left[(b-a)^{\beta-\alpha} \int_{a}^{b}\left(D_{a}^{\beta} f(y)\right)^{s} d y-(b-a)^{(\beta-\alpha)(1-s)}(\Gamma(\beta-\alpha+1))^{s} \int_{a}^{b}\left(D_{a}^{\alpha} f(x)\right)^{s} d x\right] \\
& =\frac{(b-a)^{(\beta-\alpha)(1-s)}}{s(s-1)}\left[(b-a)^{(\beta-\alpha) s} \int_{a}^{b}\left(D_{a}^{\beta} f(y)\right)^{s} d y-(\Gamma(\beta-\alpha+1))^{s} \int_{a}^{b}\left(D_{a}^{\alpha} f(x)\right)^{s} d x\right] \\
& =H_{3}(s) .
\end{aligned}
$$

It follows (2.7).

Now we define Canavati-type fractional derivative ( $v$-fractional derivative of $f$ ), for details see [1, p. 446]. We consider

$$
C^{v}([a, b])=\left\{f \in C^{n}([a, b]): I_{a+}^{n-v+1} f^{(n)} \in C^{1}([a, b])\right\},
$$

$v>0, n=[v]$. Let $f \in C^{v}([a, b])$. We define the generalized $v$-fractional derivative of $f$ over $[a, b]$ as

$$
D_{a}^{v} f=\left(I_{a+}^{n-v+1} f^{(n)}\right)^{\prime},
$$

the derivative with respect to $x$.

Lemma 2.7. Let $v \geq \gamma+1$, where $\gamma \geq 0$ and $f \in C^{v}([a, b])$. Assume $f^{(i)}(a)=0, i=0,1, \ldots,[v]-1$. Then

$$
\left(D_{a}^{\gamma} f\right)(x)=\frac{1}{\Gamma(v-\gamma)} \int_{a}^{x}(x-t)^{v-\gamma-1}\left(D_{a}^{v} f\right)(t) d t
$$

for all $x \in[a, b]$.

In the following theorem, we will construct new inequality for the Canavati-type fractional derivative.

Theorem 2.8. Let $s>1$ and assumptions in Lemma 2.7 be satisfied, $D_{a}^{\gamma} f$ denotes the Canavatitype fractional derivative of $f$ and $\xi_{4}: \mathbb{R} \rightarrow[0, \infty)$. Then the following inequality holds:

$$
\xi_{4}(s) \leq H_{4}(s)
$$


where

and

$$
\xi_{4}(s)=\frac{1}{s(s-1)}\left[\int_{a}^{b}(b-y)^{v-\gamma}\left(D_{a}^{v} f(y)\right)^{s} d y-\int_{a}^{b}(x-a)^{v-\gamma}\left(\frac{\Gamma(v-\gamma+1)}{(x-a)^{v-\gamma}} D_{a}^{\gamma} f(x)\right)^{s} d x\right]
$$

$$
H_{4}(s)=\frac{(b-a)^{(v-\gamma)(1-s)}}{s(s-1)}\left[(b-a)^{(v-\gamma) s} \int_{a}^{b}\left(D_{a}^{v} f(y)\right)^{s} d y-(\Gamma(v-\gamma+1))^{s} \int_{a}^{b}\left(D_{a}^{\gamma} f(x)\right)^{s} d x\right] .
$$

Proof. Applying Theorem 2.2 with $\Omega_{1}=\Omega_{2}=(a, b), d \mu_{1}(x)=d x, d \mu_{2}(y)=d y$,

$$
k(x, y)= \begin{cases}\frac{(x-y)^{v-\gamma-1}}{\Gamma(v-\gamma)}, & a \leq y \leq x \\ 0, & x<y \leq b\end{cases}
$$

we get that $K(x)=\frac{(x-a)^{v-\gamma}}{\Gamma(v-\gamma+1)}$. Replace $f$ by $D_{a}^{v} f$. Then the equation (2.2) becomes

$$
\xi_{4}(s)=\int_{a}^{b} v(y) \phi_{s}\left(D_{a}^{v} f(y)\right) d y-\int_{a}^{b} u(x) \phi_{s}\left(\frac{\Gamma(v-\gamma+1)}{(x-a)^{v-\gamma}} D_{a}^{\gamma} f(x)\right) d x .
$$

For particular weight function $u(x)=(x-a)^{v-\gamma}$, we get $v(y)=(b-y)^{v-\gamma}$ and take $\phi_{s}(x)=$ $\frac{x^{s}}{s(s-1)}, x \in \mathbb{R}^{+}$. We obtain that

$$
\begin{aligned}
\xi_{4}(s) & =\frac{1}{s(s-1)}\left[\int_{a}^{b}(b-y)^{v-\gamma}\left(D_{a}^{v} f(y)\right)^{s} d y-\int_{a}^{b}(x-a)^{v-\gamma}\left(\frac{\Gamma(v-\gamma+1)}{(x-a)^{v-\gamma}} D_{a}^{\gamma} f(x)\right)^{s} d x\right] \\
& \leq \frac{1}{s(s-1)}\left[(b-a)^{v-\gamma} \int_{a}^{b}\left(D_{a}^{v} f(y)\right)^{s} d y-(b-a)^{(v-\gamma)(1-s)}(\Gamma(v-\gamma+1))^{s} \int_{a}^{b}\left(D_{a}^{\gamma} f(x)\right)^{s} d x\right] \\
& =\frac{(b-a)^{(v-\gamma)(1-s)}}{s(s-1)}\left[(b-a)^{(v-\gamma) s} \int_{a}^{b}\left(D_{a}^{v} f(y)\right)^{s} d y-(\Gamma(v-\gamma+1))^{s} \int_{a}^{b}\left(D_{a}^{\gamma} f(x)\right)^{s} d x\right] \\
& =H_{4}(s)
\end{aligned}
$$

and (2.9) follows.

Next, we define Caputo fractional derivative, for details see [1, p. 449]. First, let $A C([a, b])$ be space of all absolutely continuous function on $[a, b]$. By $A C^{n}([a, b])$ we denote the space of all functions $g \in C^{n-1}([a, b])$ with $g^{(n-1)} \in A C([a, b])$.

Let $\alpha \geq 0, n=\lceil\alpha\rceil, g \in A C^{n}([a, b])$. The Caputo fractional derivative is given by

$$
D_{* a}^{\alpha} g(t)=\frac{1}{\Gamma(n-\alpha)} \int_{a}^{x} \frac{g^{(n)}(y)}{(x-y)^{\alpha-n+1}} d y,
$$

for all $x \in[a, b]$. The above function exists almost everywhere for $x \in[a, b]$.

Using the above definition, we will prove the following result as a special case of Theorem 2.2 to construct new inequality for the Caputo fractional derivative. 
Theorem 2.9. Let $s>1$ and $D_{* a}^{\gamma} f$ denotes the Caputo fractional derivative of $f$ and $\xi_{5}: \mathbb{R} \rightarrow$ $[0, \infty)$. Then the following inequality holds true:

$$
\xi_{5}(s) \leq H_{5}(s)
$$

where

$$
\xi_{5}(s)=\frac{1}{s(s-1)}\left[\int_{a}^{b}(b-y)^{n-\alpha}\left(f^{(n)}(y)\right)^{s} d y-\int_{a}^{b}(x-a)^{n-\alpha}\left(\frac{\Gamma(n-\alpha+1)}{(x-a)^{n-\alpha}} D_{* a}^{\alpha} f(x)\right)^{s} d x\right]
$$

and

$$
H_{5}(s)=\frac{(b-a)^{(n-\alpha)(1-s)}}{s(s-1)}\left[(b-a)^{(n-\alpha) s} \int_{a}^{b}\left(f^{(n)}(y)\right)^{s} d y-(\Gamma(n-\alpha+1))^{s} \int_{a}^{b}\left(D_{* a}^{\alpha} f(x)\right)^{s} d x\right] .
$$

Proof. Applying Theorem 2.2 with $\Omega_{1}=\Omega_{2}=(a, b), d \mu_{1}(x)=d x, d \mu_{2}(y)=d y$,

$$
k(x, y)= \begin{cases}\frac{(x-y)^{n-\alpha-1}}{\Gamma(n-\alpha)}, & a \leq y \leq x \\ 0, & x<y \leq b\end{cases}
$$

we get that $K(x)=\frac{(x-a)^{n-\alpha}}{\Gamma(n-\alpha+1)}$. Replace $f$ by $f^{(n)}$, Then the equation (2.2) becomes

$$
\xi_{5}(s)=\int_{a}^{b} v(y) \phi_{s}\left(f^{(n)}(y)\right) d y-\int_{a}^{b} u(x) \phi_{s}\left(\frac{\Gamma(n-\alpha+1)}{(x-a)^{n-\alpha}} D_{* a}^{\alpha} f(x)\right) d x .
$$

For particular weight function $u(x)=(x-a)^{n-\alpha}$, we get $v(y)=(b-y)^{n-\alpha}$, and we take $\phi_{s}(x)$ $=\frac{x^{s}}{s(s-1)}, x \in \mathbb{R}^{+}$and after a simple calculation (2.11) becomes (2.10).

We continue with the following lemma that is given in [1, p. 450].

Lemma 2.10. Let $\alpha \geq \gamma+1, \gamma>0$ and $n=\lceil\alpha\rceil$. Assume $f \in A C^{n}([a, b])$ such that $f^{(k)}(a)=0, k=$ $0,1, \ldots, n-1$, and $D_{* a}^{\alpha} f \in L_{\infty}(a, b)$. Then $D_{* a}^{\gamma} f \in C([a, b])$, and

$$
D_{* a}^{\gamma} f(x)=\frac{1}{\Gamma(\alpha-\gamma)} \int_{a}^{x}(x-y)^{\alpha-\gamma-1} D_{* a}^{\alpha} f(y) d y
$$

for all $a \leq x \leq b$.

Theorem 2.11. Let $s>1$ and assumptions in Lemma 2.10 be satisfied. $D_{* a}^{\alpha} f$ denotes the Caputo fractional derivative of $f$ and $\xi_{6}: \mathbb{R} \rightarrow[0, \infty)$. Then the following inequality holds:

$$
\xi_{6}(s) \leq H_{6}(s)
$$

where

$$
\begin{aligned}
& \qquad \begin{aligned}
\xi_{6}(s) & =\frac{1}{s(s-1)}\left[\int_{a}^{b}(b-y)^{\alpha-\gamma}\left(D_{* a}^{\alpha} f(y)\right)^{s} d y-\int_{a}^{b}(x-a)^{\alpha-\gamma}\left(\frac{\Gamma(\alpha-\gamma+1)}{(x-a)^{\alpha-\gamma}} D_{* a}^{\gamma} f(x)\right)^{s} d x\right] \\
\text { and } & H_{6}(s)=\frac{(b-a)^{(\alpha-\gamma)(1-s)}}{s(s-1)}\left[(b-a)^{(\alpha-\gamma) s} \int_{a}^{b}\left(D_{* a}^{\alpha} f(y)\right)^{s} d y-(\Gamma(\alpha-\gamma+1))^{s} \int_{a}^{b}\left(D_{* a}^{\gamma} f(x)\right)^{s} d x\right] .
\end{aligned}
\end{aligned}
$$


Proof. Applying Theorem 2.2 with $\Omega_{1}=\Omega_{2}=(a, b), d \mu_{1}(x)=d x, d \mu_{2}(y)=d y$,

$$
k(x, y)= \begin{cases}\frac{(x-y)^{\alpha-\gamma-1}}{\Gamma(\alpha-\gamma)}, & a \leq y \leq x \\ 0, & x<y \leq b\end{cases}
$$

we get that $K(x)=\frac{(x-a)^{\alpha-\gamma}}{\Gamma(\alpha-\gamma+1)}$. Replace $f$ by $D_{* a}^{\alpha} f$. Then the equation (2.2) becomes

$$
\xi_{6}(s)=\int_{a}^{b} v(y) \phi_{s}\left(D_{* a}^{\alpha} f(y)\right) d y-\int_{a}^{b} u(x) \phi_{s}\left(\frac{\Gamma(\alpha-\gamma+1)}{(x-a)^{\alpha-\gamma}} D_{* a}^{\gamma} f(x)\right) d x .
$$

For particular weight function $u(x)=(x-a)^{\alpha-\gamma}$, we obtain $v(y)=(b-y)^{\alpha-\gamma}$ and we choose $\phi_{s}(x)=\frac{x^{s}}{s(s-1)}, x \in \mathbb{R}^{+}$and after a simple calculation (2.13) becomes (2.12).

We continue with definitions and some properties of the fractional integrals of a function $f$ with respect to given function g. For details see e.g. [11, p.99]:

Let $(a, b),-\infty \leq a<b \leq \infty$ be a finite or infinite interval of the real line $\mathbb{R}$ and $\alpha>0$. Also let $g$ be an increasing function on $(a, b]$ and $g^{\prime}$ be a continuous function on $(a, b)$. The leftand right-sided fractional integrals of a function $f$ with respect to another function $g$ in $[a, b]$ are given by

and

$$
\left(I_{a+; g}^{\alpha} f\right)(x)=\frac{1}{\Gamma(\alpha)} \int_{a}^{x} \frac{g^{\prime}(t) f(t) d t}{[g(x)-g(t)]^{1-\alpha}}, x>a
$$

$$
\left(I_{b-; g}^{\alpha} f\right)(x)=\frac{1}{\Gamma(\alpha)} \int_{x}^{b} \frac{g^{\prime}(t) f(t) d t}{[g(t)-g(x)]^{1-\alpha}}, x<b,
$$

respectively.

Our first result involving fractional integral of $f$ with respect to another increasing function $g$ is given in the following Theorem.

Theorem 2.12. Let $s>1, \alpha>0, g$ be increasing function on $(a, b]$ such that $g^{\prime}$ be continuous on $(a, b), I_{a_{+} ; g}^{\alpha} f$ denotes the left sided fractional integral of $f$ with respect to another increasing function $g$ and $\xi_{7}: \mathbb{R} \rightarrow[0, \infty)$. Then the following inequality holds:

$$
\xi_{7}(s) \leq H_{7}(s)
$$

where

$$
\begin{aligned}
\xi_{7}(s)= & \frac{1}{s(s-1)}\left[\int_{a}^{b} g^{\prime}(y)(g(b)-g(y))^{\alpha}(f(y))^{s} d y\right. \\
& \left.-\int_{a}^{b} g^{\prime}(x)(g(x)-g(a))^{\alpha}\left(\frac{\Gamma(\alpha+1)}{(g(x)-g(a))^{\alpha}} I_{a_{+} ; g}^{\alpha} f(x)\right)^{s} d x\right]
\end{aligned}
$$

and 


$$
\begin{aligned}
H_{7}(s)= & \frac{(g(b)-g(a))^{\alpha(1-s)}}{s(s-1)}\left[(g(b)-g(a))^{\alpha s} \int_{a}^{b} f^{s}(y) g^{\prime}(y) d y\right. \\
& \left.-(\Gamma(\alpha+1))^{s} \int_{a}^{b}\left(I_{a_{+} ; g}^{\alpha} f(x)\right)^{s} g^{\prime}(x) d x\right] .
\end{aligned}
$$

Proof. Applying Theorem 2.2 with $\Omega_{1}=\Omega_{2}=(a, b), d \mu_{1}(x)=d x, d \mu_{2}(y)=d y$,

$$
k(x, y)= \begin{cases}\frac{g^{\prime}(y)}{\Gamma(\alpha)(g(x)-g(y))^{1-\alpha}}, & a \leq y \leq x \\ 0, & x<y \leq b\end{cases}
$$

we get that $K(x)=\frac{1}{\Gamma(\alpha+1)}(g(x)-g(a))^{\alpha}$. Then the equation (2.2) becomes

$$
\xi_{7}(s)=\int_{a}^{b} v(y) \phi_{s}(f(y)) d y-\int_{a}^{b} u(x) \phi_{s}\left(\frac{\Gamma(\alpha+1)}{(g(x)-g(a))^{\alpha}} I_{a_{+} ; g}^{\alpha} f(x)\right) d x .
$$

For particular weight function $u(x)=g^{\prime}(x)(g(x)-g(a))^{\alpha}$, we obtain $v(y)=g^{\prime}(y)(g(b)-g(y))^{\alpha}$ and take $\phi_{s}(x)=\frac{x^{s}}{s(s-1)}, x \in \mathbb{R}^{+}$. So after small calculations (2.15) becomes (2.14).

Remark 2.13. If $g(x)=x$, then $I_{a_{+} ; x}^{\alpha} f(x)$ reduces to $I_{a_{+}}^{\alpha} f(x)$ left-sided Riemann-Liouville fractional integral and (2.14) becomes (2.4).

Similar result can be obtained for the right sided fractional integral of $f$ with respect to another increasing function $g$, but here we omit the details.

Now we present definitions and some properties of the Erdélyi-Kober type fractional integrals. Some of these definitions and results were presented in Samko et al. in [17].

Let $(a, b),(0 \leq a<b \leq \infty)$ be a finite or infinite interval of the half-axis $\mathbb{R}^{+}$. Also let $\alpha>0, \sigma>0$, and $\eta \in \mathbb{R}$. We consider the left- and right-sided integrals of order $\alpha \in \mathbb{R}$ defined by

and

$$
\left(I_{a_{+} ; \sigma ; \eta}^{\alpha} f\right)(x)=\frac{\sigma x^{-\sigma(\alpha+\eta)}}{\Gamma(\alpha)} \int_{a}^{x} \frac{t^{\sigma \eta+\sigma-1} f(t) d t}{\left(x^{\sigma}-t^{\sigma}\right)^{1-\alpha}}, x>a
$$

$$
\left(I_{b_{-} ; \sigma ; \eta}^{\alpha} f\right)(x)=\frac{\sigma x^{\sigma \eta}}{\Gamma(\alpha)} \int_{x}^{b} \frac{t^{\sigma(1-\eta-\alpha)-1} f(t) d t}{\left(t^{\sigma}-x^{\sigma}\right)^{1-\alpha}}, \quad x<b,
$$

respectively. Integrals (2.16) and (2.17) are called the Erdélyi-Kober type fractional integrals.

Now, we give the following result.

Theorem 2.14. Let $s>1, I_{a_{+} ; \sigma ; \eta}^{\alpha} f$ denotes the Erdélyi-Kober type fractional integral of $f,{ }_{2} F_{1}(a, b ; c ; z)$ denotes the hypergeometric function and $\xi_{8}: \mathbb{R} \rightarrow[0, \infty)$. Then the following inequality holds:

$$
\xi_{8}(s) \leq H_{8}(s),
$$


where

$$
\begin{aligned}
\xi_{8}(s)= & \int_{a}^{b} y^{\sigma-1}\left(b^{\sigma}-y^{\sigma}\right)^{\alpha}{ }_{2} F_{1}(y) f^{s}(y) d y \\
& -\int_{a}^{b} x^{\sigma-1}\left(x^{\sigma}-a^{\sigma}\right)^{\alpha}{ }_{2} F_{1}(x)\left(\frac{\Gamma(\alpha+1)}{\left(1-\left(\frac{a}{x}\right)^{\sigma}\right)^{\alpha}{ }_{2} F_{1}(x)} I_{a_{+} ; \sigma ; \eta}^{\alpha} f(x)\right)^{s} d x
\end{aligned}
$$

and

$$
\begin{aligned}
H_{8}(s)= & \frac{\left(b^{\sigma}-a^{\sigma}\right)^{\alpha(1-s)}}{s(s-1)}\left[\left(b^{\sigma}-a^{\sigma}\right)^{\alpha s} b^{\sigma-1} \int_{a}^{b}{ }_{2} F_{1}(y) f^{s}(y) d y\right. \\
& \left.\left.-a^{\sigma-1+\alpha \sigma s}(\Gamma(\alpha+1))^{s} \int_{a}^{b}\left({ }_{2} F_{1}(x)\right)^{1-s} I_{a_{+} ; \sigma ; \eta}^{\alpha} f(x)\right)^{s} d x\right], \\
{ }_{2} F_{1}(x)= & { }_{2} F_{1}\left(-\eta, \alpha ; \alpha+1 ; 1-\left(\frac{a}{x}\right)^{\sigma}\right) \text { and }{ }_{2} F_{1}(y)={ }_{2} F_{1}\left(\eta, \alpha ; \alpha+1 ; 1-\left(\frac{b}{y}\right)^{\sigma}\right) .
\end{aligned}
$$

Proof. Applying Theorem 2.2 with $\Omega_{1}=\Omega_{2}=(a, b), d \mu_{1}(x)=d x, d \mu_{2}(y)=d y$,

$$
k(x, y)= \begin{cases}\frac{1}{\Gamma(\alpha)} \frac{\sigma x^{-\sigma(\alpha+\eta)}}{\left(x^{\sigma}-y^{\sigma}\right)^{1-\alpha}} y^{\sigma \eta+\sigma-1}, & a \leq y \leq x \\ 0, & x<y \leq b\end{cases}
$$

we get that $K(x)=\frac{1}{\Gamma(\alpha+1)}\left(1-\left(\frac{a}{x}\right)^{\sigma}\right)^{\alpha}{ }_{2} F_{1}\left(-\eta, \alpha ; \alpha+1 ; 1-\left(\frac{a}{x}\right)^{\sigma}\right)$. So, the equation (2.2) becomes

$$
\xi_{8}(s)=\int_{a}^{b} v(y) \phi_{s}(f(y)) d y-\int_{a}^{b} u(x) \phi_{s}\left(\frac{\Gamma(\alpha+1)}{\left(1-\left(\frac{a}{x}\right)^{\sigma}\right)^{\alpha}{ }_{2} F_{1}(x)} I_{a_{+} ; \sigma ; \eta}^{\alpha} f(x)\right) d x .
$$

For particular weight function $u(x)=x^{\sigma-1}\left(x^{\sigma}-a^{\sigma}\right){ }_{2}{ }_{2} F_{1}(x)$, where ${ }_{2} F_{1}(x)={ }_{2} F_{1}(-\eta, \alpha ; \alpha+$ $\left.\left.1 ; 1-\left(\frac{a}{x}\right)^{\sigma}\right)\right)$, we obtain $v(y)=y^{\sigma-1}\left(b^{\sigma}-y^{\sigma}\right)^{\alpha}{ }_{2} F_{1}(y)$ where ${ }_{2} F_{1}(y)={ }_{2} F_{1}\left(\eta, \alpha ; \alpha+1 ; 1-\left(\frac{b}{y}\right)^{\sigma}\right)$. Then the equation (2.19) becomes

$$
\begin{aligned}
\xi_{8}(s)= & \int_{a}^{b} y^{\sigma-1}\left(b^{\sigma}-y^{\sigma}\right)^{\alpha}{ }_{2} F_{1}(y) \phi_{s}(f(y)) d y \\
& -\int_{a}^{b} x^{\sigma-1}\left(x^{\sigma}-a^{\sigma}\right)^{\alpha}{ }_{2} F_{1}(x) \phi_{s}\left(\frac{\Gamma(\alpha+1)}{\left(1-\left(\frac{a}{x}\right)^{\sigma}\right)^{\alpha}{ }_{2} F_{1}(x)} I_{a_{+} ; \sigma ; \eta}^{\alpha} f(x)\right) d x .
\end{aligned}
$$

We choose $\phi_{s}(x)=\frac{x^{s}}{s(s-1)}, x \in \mathbb{R}^{+}$, that is,

$$
\begin{aligned}
\xi_{8}(s)= & \frac{1}{s(s-1)}\left[\int_{a}^{b} y^{\sigma-1}\left(b^{\sigma}-y^{\sigma}\right)^{\alpha}{ }_{2} F_{1}(y) f^{s}(y) d y\right. \\
& \left.-\int_{a}^{b} x^{\sigma-1}\left(x^{\sigma}-a^{\sigma}\right)^{\alpha}{ }_{2} F_{1}(x)\left(\frac{\Gamma(\alpha+1)}{\left(1-\left(\frac{a}{x}\right)^{\sigma}\right)^{\alpha}{ }_{2} F_{1}(x)} I_{a_{+} ; \sigma ; \eta}^{\alpha} f(x)\right)^{s} d x\right] \\
\leq & \frac{1}{s(s-1)}\left[b^{\sigma-1}\left(b^{\sigma}-a^{\sigma}\right)^{\alpha} \int_{a}^{b}{ }_{2} F_{1}(y) f^{s}(y) d y\right. \\
& \left.\left.-a^{\sigma-1+\alpha \sigma s}\left(b^{\sigma}-a^{\sigma}\right)^{\alpha(1-s)}(\Gamma(\alpha+1))^{s} \int_{a}^{b}{ }_{2} F_{1}(x)\right)^{1-s}\left(I_{a_{+} ; \sigma ; \eta}^{\alpha} f(x)\right)^{s} d x\right]
\end{aligned}
$$




$$
\begin{aligned}
& =\frac{\left(b^{\sigma}-a^{\sigma}\right)^{\alpha(1-s)}}{s(s-1)}\left[\left(b^{\sigma}-a^{\sigma}\right)^{\alpha s} b^{\sigma-1} \int_{a}^{b}{ }_{2} F_{1}(y) f^{s}(y) d y\right. \\
& \left.-a^{\sigma-1+\alpha \sigma s}(\Gamma(\alpha+1))^{s} \int_{a}^{b}\left({ }_{2} F_{1}(x)\right)^{1-s}\left(I_{a_{+} ; \sigma ; \eta}^{\alpha} f(x)\right)^{s} d x\right] \\
& =H_{8}(s)
\end{aligned}
$$

it follows (2.18).

Remark 2.15. Similar result can be obtained for the right sided Erdélyi-Kober type fractional integrals, but here we omit the details.

Now we continue with the definition of Hadamard-type fractional integrals.

Let $[a, b]$ be finite or infinite interval of $\mathbb{R}^{+}$and $\alpha>0$. The left and right-sided Hadamardtype fractional integrals of order $\alpha>0$ is given by

and

$$
\left(J_{a_{+}}^{\alpha} f\right)(x)=\frac{1}{\Gamma(\alpha)} \int_{a}^{x}\left(\log \frac{x}{y}\right)^{\alpha-1} \frac{f(y) d y}{y}, x>a
$$

$$
\left(J_{b_{-}}^{\alpha} f\right)(x)=\frac{1}{\Gamma(\alpha)} \int_{x}^{b}\left(\log \frac{y}{x}\right)^{\alpha-1} \frac{f(y) d y}{y}, x<b
$$

respectively.

The following result is about the Hadamard-type fractional integrals.

Theorem 2.16. Let $s>1, \alpha>0, J_{a_{+}}^{\alpha} f$ denotes the Hadamard-type fractional integrals of $f$ and $\xi_{9}: \mathbb{R} \rightarrow[0, \infty)$. Then the following inequality holds:

$$
\xi_{9}(s) \leq H_{9}(s)
$$

where

$$
\xi_{9}(s)=\frac{1}{s(s-1)}\left[\int_{a}^{b} \frac{(\log b-\log y)^{\alpha}}{y} f^{s}(y) d y-\int_{a}^{b} \frac{(\log x-\log a)^{\alpha}}{x}\left(\frac{\Gamma(\alpha+1)}{(\log x-\log a)^{\alpha}}\left(J_{a_{+}}^{\alpha} f(x)\right)\right)^{s} d x\right]
$$

and

$H_{9}(s)=\frac{1}{s(s-1)} \frac{(\log b-\log a)^{\alpha(1-s)}}{a b}\left[b(\log b-\log a)^{\alpha s} \int_{a}^{b} f^{s}(y) d y-a(\Gamma(\alpha+1))^{s} \int_{a}^{b}\left(J_{a_{+}}^{\alpha} f(x)\right)^{s} d x\right]$.

Proof. Applying Theorem 2.2 with $\Omega_{1}=\Omega_{2}=(a, b), d \mu_{1}(x)=d x, d \mu_{2}(y)=d y$,

$$
k(x, y)= \begin{cases}\frac{(\log x-\log y)^{\alpha-1}}{y \Gamma(\alpha)}, & a \leq y \leq x, \\ 0, & x<y \leq b\end{cases}
$$

we get that $K(x)=\frac{1}{\Gamma(\alpha+1)}(\log x-\log a)^{\alpha}$. Then the equation (2.2) becomes

$$
\xi_{9}(s)=\int_{a}^{b} v(y) \phi_{s}(f(y)) d y-\int_{a}^{b} u(x) \phi_{s}\left(\frac{\Gamma(\alpha+1)}{(\log x-\log a)^{\alpha}} J_{a_{+}}^{\alpha} f(x)\right) d x .
$$


For particular weight function $u(x)=\frac{(\log x-\log a)^{\alpha}}{x}$, we obtain $v(y)=\frac{(\log b-\log y)^{\alpha}}{y}$. So (2.21) becomes

$$
\begin{aligned}
\xi_{9}(s)= & \int_{a}^{b} \frac{(\log b-\log y)^{\alpha}}{y} \phi_{s}(f(y)) d y \\
& -\int_{a}^{b} \frac{(\log x-\log a)^{\alpha}}{x} \phi_{s}\left(\frac{\Gamma(\alpha+1)}{(\log x-\log a)^{\alpha}} J_{a_{+}}^{\alpha} f(x)\right) d x,
\end{aligned}
$$

We choose $\phi_{s}(x)=\frac{x^{s}}{s(s-1)}, x \in \mathbb{R}^{+}$, that is

$$
\begin{aligned}
\xi_{9}(s)= & \frac{1}{s(s-1)}\left[\int_{a}^{b} \frac{(\log b-\log y)^{\alpha}}{y} f^{s}(y) d y\right. \\
& \left.-\int_{a}^{b} \frac{(\log x-\log a)^{\alpha}}{x}\left(\frac{\Gamma(\alpha+1)}{(\log x-\log a)^{\alpha}}\left(J_{a_{+}}^{\alpha} f(x)\right)\right)^{s} d x\right] \\
\leq & \frac{1}{s(s-1)}\left[\frac{(\log b-\log a)^{\alpha}}{a} \int_{a}^{b} f^{s}(y) d y\right. \\
& \left.-\Gamma(\alpha+1))^{s} \frac{(\log b-\log a)^{\alpha(1-s)}}{b} \int_{a}^{b}\left(J_{a_{+}}^{\alpha} f(x)\right)^{s} d x\right] \\
= & \frac{1}{s(s-1)} \frac{(\log b-\log a)^{\alpha(1-s)}}{a b}\left[b(\log b-\log a)^{\alpha s} \int_{a}^{b} f^{s}(y) d y\right. \\
& \left.-a(\Gamma(\alpha+1))^{s} \int_{a}^{b}\left(J_{a_{+}}^{\alpha} f(x)\right)^{s} d x\right] \\
= & H_{9}(s)
\end{aligned}
$$

it follows (2.20).

Remark 2.17. Notice that Hadamard fractional integrals of order $\alpha$ are special case of the leftand right-sided fractional integrals of a function $f$ with respect to another function $g(x)=$ $\log (x)$ in $[a, b]$ where $0 \leq a<b \leq \infty$.

In the following theorem we prove the three different cases for the above results.

Theorem 2.18. For $i=1, \ldots, 9$ the following inequalities hold true:
(i). $\left[\xi_{i}(p)\right]^{\frac{q-r}{q-p}}\left[\xi_{i}(q)\right]^{\frac{r-p}{q-p}} \leq H_{i}(r)$
(ii). $\left[\xi_{i}(r)\right]^{\frac{p-q}{p-r}}\left[\xi_{i}(p)\right]^{\frac{q-r}{p-r}} \leq H_{i}(q)$
(iii). $\xi_{i}(p) \leq\left[H_{i}(r)\right]^{\frac{q-p}{q-r}}\left[H_{i}(q)\right]^{\frac{p-r}{q-r}}$

for every choice $p, q, r \in \mathbb{R}$, such that $1<r<p<q$.

Proof. We will prove this theorem just in case $i=1$, since all other case are proved analogous. 
(i). Since the function $\xi_{1}$ is exponentially convex, it is also log-convex. Then for $1<r<p<$ $q, r, p, q \in \mathbb{R},(2.3)$ can be written as

$$
\left[\xi_{1}(p)\right]^{q-r}\left[\xi_{1}(q)\right]^{r-p} \leq\left[\xi_{1}(r)\right]^{q-p} .
$$

This implies that

$$
\begin{aligned}
{\left[\xi_{1}(p)\right]^{\frac{q-r}{q-p}}\left[\xi_{1}(q)\right]^{\frac{r-p}{q-p}} } & \leq \frac{(b-a)^{\alpha(1-r)}}{r(r-1)}\left[(b-a)^{\alpha r} \int_{a}^{b} f^{r}(y) d y-(\Gamma(\alpha+1))^{r} \int_{a}^{b}\left(I_{a^{+}}^{\alpha} f(x)\right)^{r} d x\right] \\
& =H_{1}(r) .
\end{aligned}
$$

It follows (2.22).

(ii). Now (2.3) can be written as,

$$
\left[\xi_{1}(r)\right]^{p-q}\left[\xi_{1}(p)\right]^{q-r} \leq\left[\xi_{1}(q)\right]^{p-r} .
$$

This implies that

$$
\begin{aligned}
{\left[\xi_{1}(r)\right]^{\frac{p-q}{p-r}}\left[\xi_{1}(p)\right]^{\frac{q-r}{p-r}} } & \leq \frac{(b-a)^{\alpha(1-q)}}{q(q-1)}\left[(b-a)^{\alpha q} \int_{a}^{b} f^{q}(y) d y-(\Gamma(\alpha+1))^{q} \int_{a}^{b}\left(I_{a^{+}}^{\alpha} f(x)\right)^{q} d x\right] \\
& =H_{1}(q) .
\end{aligned}
$$

It follows (2.23).

(iii). The (2.3) can be written as,

$$
\begin{aligned}
& {\left[\xi_{1}(p)\right]^{\frac{q-r}{p-r}} \leq\left[\xi_{1}(r)\right]^{\frac{q-p}{p-r}} \xi_{1}(q),} \\
& {\left[\xi_{1}(p)\right]^{\frac{q-r}{p-r}} \leq\left[\xi_{1}(r)\right]^{\frac{q-p}{p-r}} H_{1}(q) .}
\end{aligned}
$$

This implies that

$$
\xi_{1}(p) \leq\left[H_{1}(r)\right]^{\frac{q-p}{q-r}}\left[H_{1}(q)\right]^{\frac{p-r}{q-r}}
$$

It follows (2.24).

\section{Mean value theorems and Cauchy means}

Now we will give the mean value theorems and means of Cauchy type for different fractional integrals and fractional derivatives. For this purpose we define a notation

$$
\xi_{i}(s):=\xi_{i}\left(\nu, \phi_{s}(f(y)) ; u, \phi_{s}\left(A_{k} f(x)\right), \text { for }(i=1, \ldots, 9)\right.
$$

where $A_{k} f$ and $v$ are defined by (1.2) and (1.4) respectively.

The following theorems are given in [4]. Such type of results are also give in [18] and [19]. 
Theorem 3.1. Let $\left(\Omega_{1}, \Sigma_{1}, \mu_{1}\right),\left(\Omega_{2}, \Sigma_{2}, \mu_{2}\right)$ be measure spaces with $\sigma$-finite measures and $u$ : $\Omega_{1} \rightarrow \mathbb{R}$ be a weight function. Let I be compact interval of $\mathbb{R}, h \in C^{2}(I)$, and $f: \Omega_{2} \rightarrow \mathbb{R} a$ measurable function such that Im $f \subseteq I$. Then there exists $\eta \in I$ such that

$$
\begin{aligned}
& \int_{\Omega_{2}} v(y) h(f(y)) d \mu_{2}(y)-\int_{\Omega_{1}} u(x) h\left(A_{k} f(x)\right) d \mu_{1}(x) \\
& \quad=\frac{h^{\prime \prime}(\eta)}{2}\left[\int_{\Omega_{2}} v(y) f^{2}(y) d \mu_{2}(y)-\int_{\Omega_{1}} u(x)\left(A_{k} f(x)\right)^{2} d \mu_{1}(x)\right],
\end{aligned}
$$

where $A_{k} f$ and $v$ are defined by (1.2) and (1.4) respectively.

Theorem 3.2. Assume that all conditions of Theorem 3.1 are satisfied. Let I be a compact interval in $\mathbb{R}$ and $g, h \in C^{2}(I)$ such that $h^{\prime \prime}(x) \neq 0$ for every $x \in I$. Let $f: \Omega_{2} \rightarrow \mathbb{R}$ be a measurable function such that $\operatorname{Im} f \subseteq I$ and

$$
\int_{\Omega_{2}} v(y) h(f(y)) d \mu_{2}(y)-\int_{\Omega_{1}} u(x) h\left(A_{k} f(x)\right) d \mu_{1}(x) \neq 0 .
$$

Then there exists $\eta \in I$ such that it holds

$$
\frac{g^{\prime \prime}(\eta)}{h^{\prime \prime}(\eta)}=\frac{\int_{\Omega_{2}} v(y) g(f(y)) d \mu_{2}(y)-\int_{\Omega_{1}} u(x) g\left(A_{k} f(x)\right) d \mu_{1}(x)}{\int_{\Omega_{2}} v(y) h(f(y)) d \mu_{2}(y)-\int_{\Omega_{1}} u(x) h\left(A_{k} f(x)\right) d \mu_{1}(x)} .
$$

We will give some special cases of Theorems 3.1 and 3.2 for different fractional integrals and fractional derivatives.

Theorem 3.3. Let $u$ be weight functions on $(a, b), A_{k} f(x)$ be defined in (1.2) and $v$ be defined in (1.4). Let I be a compact interval of $\mathbb{R}, h \in C^{2}(I)$ and $\xi_{i}: \mathbb{R} \rightarrow[0, \infty)$. Then there exists $\eta_{i} \in I$ such that

$$
\xi_{i}\left(\nu, h(f(y)) ; u, h\left(A_{k} f(x)\right)\right)=\frac{h^{\prime \prime}\left(\eta_{i}\right)}{2} \xi_{i}\left(\nu,(f(y))^{2} ; u,\left(A_{k} f(x)\right)^{2}\right), \text { for }(i=1, \ldots, 9) .
$$

Theorem 3.4. Let $u$ be weight functions on $(a, b), A_{k} f(x)$ be defined in (1.2) and $v$ be defined in (1.4). Let I be a compact interval of $\mathbb{R}, g, h \in C^{2}(I)$ such that $h^{\prime \prime}(x) \neq 0$ for every $x \in I, \xi_{i}: \mathbb{R} \rightarrow$ $[0, \infty)$ and

$$
\xi_{i}\left(\nu, h(f(y)) ; u, h\left(A_{k} f(x)\right)\right) \neq 0 .
$$

Then there exists $\eta_{i} \in I$ such that

$$
\frac{g^{\prime \prime}\left(\eta_{i}\right)}{h^{\prime \prime}\left(\eta_{i}\right)}=\frac{\xi_{i}\left(\nu, g(f(y)) ; u, g\left(A_{k} f(x)\right)\right)}{\xi_{i}\left(\nu, h(f(y)) ; u, h\left(A_{k} f(x)\right)\right)}, \text { for } \quad(i=1, \ldots, 9)
$$

If we apply Theorem 3.4 with $g(x)=\frac{x^{p}}{p(p-1)}, h(x)=\frac{x^{s}}{s(s-1)}, p \neq s, p, s \neq 0,1$, we get the following result. 
Corollary 3.5. Let $u$ be weight functions on $(a, b), A_{k} f(x)$ be defined in (1.2) and $v$ be defined in (1.4). Let I be a compact interval of $\mathbb{R}^{+}, \xi_{i}: \mathbb{R} \rightarrow[0, \infty),(i=1, \ldots, 9)$, then for $p \neq s, p, s \neq 1$, there exist $\eta_{i} \in I$ such that

$$
\eta_{i}^{p-s}=\frac{\xi_{i}(p)}{\xi_{i}(s)}=\frac{s(s-1)}{p(p-1)} \frac{\xi_{i}\left(\nu, f^{p}(y) ; u,\left(A_{k} f(x)\right)^{p}\right)}{\xi_{i}\left(\nu, f^{s}(y) ; u,\left(A_{k} f(x)\right)^{s}\right)} .
$$

Remark 3.6. Since $g^{\prime \prime}(x)=x^{p-2}$ and $h^{\prime \prime}(x)=x^{s-2}, \frac{g^{\prime \prime}}{h^{\prime \prime}}$ are invertible. Then from (3.2), we obtain

$$
\inf _{t \in[a, b]} f(t) \leq\left(\frac{\xi_{i}(p)}{\xi_{i}(s)}\right)^{\frac{1}{p-s}} \leq \sup _{t \in[a, b]} f(t) .
$$

So,

and

$$
M_{i}^{p, s}\left(\nu, \varphi_{s}(f(y)) ; u, \varphi_{s}\left(A_{k} f(x)\right)\right)=\left(\frac{\xi_{i}(p)}{\xi_{i}(s)}\right)^{\frac{1}{p-s}}
$$

$$
M_{i}^{p, s}:=M_{i}^{p, s}\left(\nu, \varphi_{s}(f(y)) ; u, \varphi_{s}\left(A_{k} f(x)\right)\right.
$$

$p \neq s, p, s \neq 0,1$ are means. Moreover we can extend these means to excluded cases. Taking a limit we can define

$$
M_{i}^{p, s}= \begin{cases}\left(\frac{\xi_{i}\left(v, \varphi_{p}(f(y)) ; u, \varphi_{p}\left(A_{k} f(x)\right)\right)}{\xi_{i}\left(\nu, \varphi_{s}(f(y)) ; u, \varphi_{s}\left(A_{k} f(x)\right)\right)}\right)^{\frac{1}{p-s}}, & p \neq s, \\ \exp \left(\frac{1-2 s}{s(s-1)}-\frac{\xi_{i}\left(\nu, \varphi_{s}(f(y)) \varphi_{0}(f(y)) ; u, \varphi_{s}\left(A_{k} f(x)\right) \varphi_{0}\left(A_{k} f(x)\right)\right)}{\xi_{i}\left(\nu, \varphi_{s}(f(y)) ; u, \varphi_{s}\left(A_{k} f(x)\right)\right)}\right), & p=s \neq 0,1, \\ \exp \left(\frac{-\xi_{i}\left(\nu, \varphi_{1}(f(y))\left(\varphi_{0}(f(y)+2)\right)\right) ; u, \varphi_{1}\left(A_{k} f(x)\right)\left(\varphi_{0}\left(A_{k} f(x)+2\right)\right)}{2 \xi_{i}\left(\nu, f(y)+\varphi_{1}(f(y)) ; u, A_{k} f(x)+\varphi_{1}\left(A_{k} f(x)\right)\right)}\right), p=s=1 & \\ \exp \left(\frac{\xi_{i}\left(\nu,\left(2 \varphi_{0}(f(y))-\varphi_{0}^{2}(f(y))\right) ; u,\left(2 \varphi_{0}\left(A_{k} f(x)\right)-\varphi_{0}^{2}\left(A_{k} f(x)\right)\right)\right)}{2 \xi_{i}\left(\nu, 1+\varphi_{0} f(y) ; u, 1+\varphi_{0}\left(A_{k} f(x)\right)\right)}\right), & p=s=0\end{cases}
$$

In the following theorem, we prove the monotonicity of means.

Theorem 3.7. Let $r \leq s, l \leq p$, then the following inequality is valid,

$$
M_{i}^{l, r} \leq M_{i}^{p, s} \text { for } \quad i=1, \ldots, 9 .
$$

that is , the means $M_{i}^{p, s}$ are monotonic.

Proof. Since $\xi_{i}$ are exponentially convex is also log-convex, we can apply Lemma 1.4 and get (3.3). For $r=s, l=p$ we get the result by taking limit in (3.3). This completes the proof.

\section{Acknowledgements}

This research work is partially funded by Higher Education Commission of Pakistan. The research of the second and third author was supported by the Croatian Ministry of Science, Education and Sports, under the Research Grant 117-1170889-0888. 


\section{References}

[1] G. A. Anastassiou, Fractional Differentiation Inequalities, Springer Science-Businness Media, LLC, Dordrecht, the Netherlands, 2009.

[2] S. N. Bernstein, Sur les fonctions absolument monotones, Acta Math., 52 (1929), 1-66.

[3] D. E. Edmunds, V. Kokilashvili and A. Meskhi, Bounded and Compact Integral Operators, Kluwer Academic Publishers, 101, Philip Drive, Norwell, MA02061, USA.

[4] N. Elezović, K. Krulić and J. Pečarić, Bounds for Hardy type differences, Acta Mathematica Sinica, English Series, 27(4) (2011), 671-684.

[5] G. D. Handley, J. J. Koliha and J. Pečarić, Hilbert-Pachpatte type integral inequalities for fractional derivatives, Fract. Calc. Appl. Anal., 4(2001), 37-46.

[6] G. H. Hardy, Notes on some points in the integrral calculus, Messenger. Math., 47(10) (1918), 145-150.

[7] S. Iqbal, J. Pečarić and Y. Zhou, Generalization of an inequality for integral transforms with kernel and related results, J. Inequal. Appl., vol. 2010. Artical ID 948430, 2010.

[8] S. Iqbal, K. Krulić and J. Pečarić, On an inequality of H. G. Hardy, J. Inequal. Appl., vol. 2010. Artical ID 264347, 2010.

[9] J. Jakšetić and J. Pečarić, Exponential convexity method, J. Convex Anal., (accepted).

[10] S. Kaijser, L. Nikolova, L-E. Persson and A. Wedestig, Hardy type inequalities via convexity, Math. Inequal. Appl. 8(3)(2005), 403-417.

[11] A. A. Kilbas, H. M. Srivastava and J. J. Trujillo, Theory and Application of Fractinal Differential Equations, North-Holland Mathematics Sttudies, 204, Elsevier, New York-London, 2006.

[12] K. Krulić, J. Pečarić and L. E. Persson, Some new Hardy type inequalities with general kernels, Math. Inequal. Appl., 12(2009), 473-485.

[13] D. S. Mitrinović, J. Pečarić and A. M. Fink, Classical and New Inequalities in Analysis, Kluwer Academic Publisher, 1993.

[14] D. S. Mitrinović and J. E. Pečarić, On Some Inequalities for Monotone Functions, Boll. Unione. Math. Ital., (7), 5-13, 407-416, 1991.

[15] C. Niculescu and L.-E. Persson, Convex Functions and Their Applications. A Contemporary Approach, CMC Books in Mathematics, Springer, New York, 2006.

[16] J. Pečarić, F. Proschan and Y. L. Tong, Convex Functions, Partial Orderings and Statistical Applications, Academic Press, Inc. 1992.

[17] S. G. Samko, A. A. Kilbas and O. J. Marichev, Fractional Integral and Derivatives : Theory and Applications, Gordon and Breach Science Publishers, Switzerland, 1993.

[18] J. Pečarić, I. Perić and H. M. Srivastava, On the Cauchy type mean value theorems, J. Math. Anal. Appl. 306(2005), 730-739.

[19] J. J. Trujillo, M. Rivero and B. Bonila, On Riemann-Liouville generalized Taylor's formula, J. Math. Anal. Appl. 231 (1999), 255-265.

${ }^{1}$ Abdus Salam School of Mathematical Sciences, GC University Lahore, Pakistan.

E-mail: sajid_uos2000@yahoo.com

${ }^{2}$ Faculty of Textile Technology, University of Zagreb, Prilaz baruna Filipovića 28a, 10000 Zagreb, Croatia.

E-mail: kkrulic@ttf.hr

${ }^{3}$ Faculty of Textile Technology, University of Zagreb, Prilaz baruna Filipovića 28a, 10000 Zagreb, Croatia.

E-mail: pecaric@element.hr 